\title{
Aproveitamento da biomassa florestal na fabricação de briquetes
}

\section{The use of forest biomass in the manufacture of briquettes}

\author{
Angelo Fernando de Oliveira Saccol ${ }^{1}$, Carline Andréa Welter ${ }^{1}$, \\ Rossana Cortelini da Rosa ${ }^{1}$, Rodrigo Coldebella ', \\ Solon Jonas Longhi ${ }^{1}$, Jorge Antonio de Farias ${ }^{1}$, \\ Cristiane Pedrazzi ${ }^{i}$
}

\footnotetext{
${ }^{1}$ Programa de Pós-Graduação em Engenharia Florestal, Universidade Federal de Santa Maria - UFSM, Santa Maria, RS, Brasil.

e-mail: fernando.saccol@hotmail.com, carlinewelter@gmail.com, ro.cortelini@hotmail.com, rodrigocoldebella@yahoo.com.br, longhi.solon@gmail.com, fariasufsm@gmail.com, cpedrazzi@terra.com.br
}

\section{RESUMO}

A importância do emprego de fontes alternativas de energia, que sejam renováveis e provoquem menores danos ao meio ambiente, como menor poluição atmosférica e estabilidade do ciclo do carbono, traz em destaque a biomassa florestal. Nesse contexto, avaliou-se a produção de briquetes, produzidos a partir de resíduos florestais encontrados nas diversas fases de produção das fábricas de celulose, os quais foram analisados quimicamente, bem como suas propriedades físicas e mecânicas. Foram realizados dez tratamentos a partir de quatro classes de resíduos amostrados, utilizando delineamento estatístico inteiramente casualizado (DIC), com 10 repetições por tratamento. Para a análise estatística, realizou-se teste Tukey de comparação de médias e análise de componentes principais para explicar a estrutura da variância. Os tratamentos 4 e 10 apresentaram maior poder calorífico devido ao seu maior percentual de lignina, bem como obtiveram os melhores resultados de densidade energética. Quanto a densidade e a resistência mecânica, os valores encontrados foram adequados e condizentes aos citados na literatura, sendo que a última variável não diferiu estatisticamente entre os tratamentos. A análise dos componentes principais foi adequada, sendo os teores de extrativos, umidade, lignina, e a densidade energética as variáveis mais importantes na análise, com os mais elevados coeficientes da componente principal 1, a qual representa 70,9\% da variância total explicada. O tratamento 4, contendo apenas casca de Pinus taeda, se destacou pelas suas melhores características para energia.

Palavras-chave: Resíduos florestais, Análise multivariada, Eucalyptus spp., Pinus taeda

\begin{abstract}
The importance of using alternative sources of energy, which are renewable and cause less damage to the environment, such as lower atmospheric pressure and carbon cycle stability, highlights a forest biomass. In this context, the production of briquettes, produced from forest residues found in the various production phases of pulp mills, was evaluated and chemically analyzed, as well as their physical and mechanical properties. Ten treatments were performed from four sampled residue classes, their analysis was performed by a completely randomized design, with 10 repetitions per treatment. For the statistical analysis, Tukey's test of comparison of means and analysis of main components was performed to explain the structure of variance. Treatments 4 and 10 showed higher calorific power due to its higher lignin percentage, as well as obtained the best energy density results. Regarding the density and mechanical strength, the values found were adequate and consistent with the literature, and the last variable did not differ statistically among the treatments. The principal components analysis was adequate, with the contents of extractives, moisture, lignin, and energy density the most important variables in the analysis, with the highest coefficients of the main component 1 , which represents $70.9 \%$ of the total variance explained. Treatment 4, containing only Pinus Taeda bark, stood out for its best characteristics for energy.
\end{abstract}

Keywords: Forest residues, Multivariate analysis, Eucalyptus spp., Pinus taeda 


\section{INTRODUÇÃO}

A biomassa vegetal pode ser utilizada para obtenção das mais variadas formas de energia seja por conversão direta ou indireta. Como vantagens da utilização desse material em substituição aos combustíveis fósseis podemos citar a menor poluição atmosférica, além da estabilidade do ciclo de carbono. Já em comparação a outros tipos de energias renováveis, a biomassa vegetal, se destaca pela alta densidade energética e pelas facilidades de armazenamento, conversão e transporte desse material [1], sendo a segunda fonte de geração de energia predominante no Brasil [2].

As biomassas para uso energético provem de recursos florestais, seus produtos e subprodutos, que incluem basicamente resíduos lenhosos produzidos de forma sustentável [3], podendo ser obtidos de florestas cultivadas, e também em atividades que processam ou utilizam a madeira para fins não energéticos, destacando-se as indústrias de papel/celulose e moveleira, bem como as serrarias[4]. As indústrias de base florestal, como as de celulose, têm por característica a geração de um grande volume de resíduos ao longo do seu processo produtivo. O setor madeireiro apresenta um grande potencial para o aproveitamento desses resíduos, já que as perdas são inerentes ao processo produtivo, representando de 40 a $70 \%$ do volume da matéria-prima [5]. Estes resíduos (cascas, galhos, folhas, cavacos e lascas de madeira, etc) são provenientes da colheita e do beneficiamento da madeira e por muito tempo, não tiveram destinação adequada [6].

A queima de biomassa florestal em caldeiras, em muitas indústrias, como as que produzem polpa celulósica, contribuem e muitas vezes satisfazem às necessidades energéticas na linha de fibras, auxiliando na maior rentabilidade da empresa pela redução de custos em seus processos produtivos. De acordo com Foelkel [6], a matriz energética como um todo do setor de polpa e papel no Brasil registra que mais de $75 \%$ da energia consumida deriva de biomassa florestal.

Existem diferentes maneiras de aproveitamento da biomassa florestal pelas indústrias, sendo a briquetagem uma forma eficaz de aproveitar esses resíduos [7], uma vez que este processo permite o aumento do teor energético dos resíduos através do adensamento e compactação [8]. Para melhor adequação da biomassa ao processo de briquetagem como combustível, algumas alternativas podem ser utilizadas visando a melhoria do rendimento do processo, como adequação do tamanho e secagem das partículas e as condições empregadas para a prensagem do material [9].

Nesse contexto, objetivou-se nesse estudo avaliar o uso de biomassa florestal, provenientes de indústrias de papel e celulose, na produção de briquetes, visando apresentar alternativas para a geração de energia limpa, assim como um novo destino para os resíduos gerados na floresta e no pátio de madeira das empresas.

\section{MATERIAIS E MÉTODOS}

O estudo foi desenvolvido no Laboratório de Produtos Florestais do Departamento de Ciências Florestais da Universidade Federal de Santa Maria. A biomassa ou resíduos florestais foram obtidos de indústrias de papel e celulose e separados em quatro classes de acordo com a espécie, local de coleta e composição (Tabela 1).

Tabela 1: Classificação dos resíduos florestais conforme espécie, local de coleta e composição da biomassa.

\begin{tabular}{|c|c|c|c|}
\hline CLASSE & ESPÉCIE & LOCAL DE COLETA & COMPOSIÇÃO \\
\hline 1 & \multirow{3}{*}{ Eucalyptus spp. } & Pátio de madeira & Serragem e palitos de madeira \\
\hline 2 & & Floresta & Cascas e folhas \\
\hline 3 & & Área industrial & Cavacos e lascas de madeira \\
\hline 4 & Pinus taeda & Pátio de madeira & Cascas \\
\hline
\end{tabular}

Após classificados, os resíduos foram deixados secar ao ar livre, separadamente, até atingir umidade de equilíbrio de aproximadamente $12 \%$. Devido à desuniformidade do tamanho dos resíduos provenientes das empresas, foi necessário triturá-los em moinho Willey para adequar a sua granulometria [10].

Parte dos resíduos florestais triturados no moinho foi classificada para as análises químicas. Nesse caso, utilizou-se as partículas que passaram pela peneira 40 mesh, ficando retidas em peneira de 60 mesh, conforme a norma TAPPI T204 cm-97 [11]. O restante dos resíduos foi classificado em peneira de 10 mesh a fim de obter amostras com partículas homogêneas para fabricação dos briquetes. Optou-se por esta granulometria para que os resíduos tivessem maior compactação durante a fabricação dos briquetes.

No total foram realizados 10 tratamentos para a produção dos briquetes, 4 tratamentos contendo $100 \%$ de cada classe de resíduos e 6 tratamentos formulados em proporções de $50-50 \%$ de cada classe. (Tabela 2), 
com 10 repetições por tratamento. O delineamento foi o inteiramente casualizado (DIC). As variáveis analisadas foram os teores de umidade, lignina klason, extrativos totais, cinzas, o poder calorífico, densidade e resistência a compressão dos briquetes.

Tabela 2: Tratamentos empregados para a confecção dos briquetes em função das classes de resíduos florestais.

\begin{tabular}{c|c|c}
\hline TRATAMENTO & CLASSE DE RESÍDUO (CR) & PROPORÇÃO DAS CLASSES \\
\hline 1 & 1 & $100 \%$ \\
\hline 2 & 2 & $100 \%$ \\
\hline 3 & 3 & $100 \%$ \\
\hline 4 & 4 & $100 \%$ \\
\hline 5 & 1 e & $50 \%$ Classe $1: 50 \%$ Classe 2 \\
\hline 6 & 1 e 3 & $50 \%$ Classe $1: 50 \%$ Classe 3 \\
\hline 7 & 1 e 4 & $50 \%$ Classe $1: 50 \%$ Classe 4 \\
\hline 8 & 2 e 3 & $50 \%$ Classe 2:50\% Classe 3 \\
\hline 9 & 2 e 4 & $50 \%$ Classe 2 :50\% Classe 4 \\
\hline 10 & 3 e 4 & $50 \%$ Classe 3 : 50\% Classe 4 \\
\hline
\end{tabular}

A umidade das partículas foi determinada pela diferença de uma massa conhecida antes e após a secagem à $105^{\circ} \mathrm{C}$ até peso constante em estufa de secagem, com controle de temperatura, conforme metodologia descrita na norma TAPPI T210 cm-93 [12]. Ainda, foram realizadas as seguintes análises para a caracterização química dos tratamentos: teores de extrativos totais (TAPPI T264 cm-97) [13], de lignina klason (TAPPI T222 om-98) [14] e de compostos inorgânicos (TAPPI T211 om-93) [15].

O poder calorífico superior (PCS) dos tratamentos foi mensurado a partir de amostras moídas e classificadas em peneiras de 60 mesh, as quais foram colocadas em estufa a $105 \pm 5^{\circ} \mathrm{C}$ por 48 horas (até atingirem peso constante) para retirada total da umidade, sendo utilizada uma porção de $0,5 \mathrm{~g}$ da amostra completamente seca, em uma bomba calorimétrica adiabática, modelo IKA C5000. O poder calorífico útil foi obtido a partir dos valores do poder calorífico inferior (PCI), empregando as equações a seguir:

$$
\begin{aligned}
& \text { PCI }\left(\frac{\text { Kcal }}{\mathrm{kg}}\right)=\mathrm{PCS} \times(1-\mathrm{TUbu})-(600 \times \mathrm{TUbu}) \\
& \mathrm{PCU}\left(\frac{\mathrm{Kcal}}{\mathrm{kg}}\right)=\mathrm{PCI} \times \frac{(100-\mathrm{TUbu})}{100}-(6 \times \mathrm{H})
\end{aligned}
$$

Em que: $\mathrm{PCI}=$ poder calorífico inferior, $\mathrm{PCS}=$ poder calorífico superior, $\mathrm{PCU}=$ poder calorífico útil, expressos em Kcal $/ \mathrm{kg}$, TUb.u. = teor de umidade base úmida da amostra, $\mathrm{H}=$ teor de hidrogênio (\% em base seca), para o qual foi considerado 6\% de hidrogênio para todos as classes de resíduo[16].

Os briquetes foram produzidos em briquetadeira modelo LIPPEL LB-32, sob parâmetros de fabricação pré-definidos após testes preliminares. As condições empregadas foram pressão de 100 bar, temperatura de $120 \pm 5^{\circ} \mathrm{C}$, tempo de compactação e de resfriamento de 3 e 6 minutos, respectivamente. A densidade aparente dos briquetes foi determinada pelo método estereométrico, ou seja, pela relação da massa/volume em um dado teor de umidade. Por serem cilíndricos, as medições do diâmetro dos briquetes foram realizadas em três pontos (base, meio e topo), utilizando um paquímetro digital com precisão de $10^{-3} \mathrm{~mm}$. A massa do briquete foi determinada por meio de uma balança analítica com precisão de $10^{-2} \mathrm{~g}$. A densidade energética do briquetes foi calculada por meio do produto do poder calorífico útil (PCU) e da densidade aparente (DA), conforme a equação:

$$
\operatorname{DE}\left(\frac{\mathrm{Mcal}}{\mathrm{m}^{3}}\right)=\frac{\mathrm{PCU} \times \mathrm{DA}}{1000}
$$

Em que: $\mathrm{DE}=$ densidade energética expressa $\left(\mathrm{Mcal} / \mathrm{m}^{3}\right), \mathrm{PCU}=$ Poder Calorífico Útil expresso $(\mathrm{kcal} / \mathrm{kg})$; $\mathrm{DA}=$ densidade Aparente expressa $\left(\mathrm{kg} / \mathrm{m}^{3}\right)$.

O ensaio mecânico de resistência a compressão foi realizado na máquina universal de ensaio modelo EMIC, na qual os briquetes foram comprimidos no sentido perpendicular a uma velocidade de $0,3 \mathrm{~mm} \mathrm{~min}^{-1}$ até a ruptura, conforme metodologia adaptada da norma NBR ISO 11093-9 [17]. 
Para a análise estatística, os dados foram tabelados em planilha eletrônica e avaliados com auxílio do software IBM SPSS Statistics 20, sendo realizado teste de comparação de médias Tukey, com 95\% de probabilidade. Realizou-se ainda, a análise de componentes principais (ACP), a fim de explicar a estrutura de variância do vetor aleatório composto pelas características avaliadas nos briquetes, consideradas as médias das variáveis dentro de cada tratamento. A matriz utilizada foi a de correlação dos dados e as combinações lineares estabelecidas foram interpretadas por meio dos autovetores normalizados e das correlações entre as variáveis originais e as componentes principais.

\section{RESULTADOS E DISCUSSÃO}

Nas Tabelas 3 e 4 são apresentados as médias e dispersão dos valores obtidos para as características químicas e propriedades físicas e mecânicas dos briquetes produzidos, respectivamente.

Tabela 3: Valores médios (\%) e dispersão ( $\sigma$ : desvio padrão) da composição química dos tratamentos.

\begin{tabular}{|c|c|c|c|c|c|c|c|c|c|c|}
\hline \multirow[t]{2}{*}{ TRATAMENTOS } & \multicolumn{2}{|c|}{ UMIDADE } & \multicolumn{2}{|c|}{$\begin{array}{c}\text { EXTRATIVOS } \\
\text { TOTAIS }\end{array}$} & \multicolumn{3}{|c|}{ LIGNINA KLASON } & \multicolumn{3}{|c|}{ CINZAS } \\
\hline & Média & $\sigma$ & Média & $\sigma$ & Média & & $\sigma$ & Média & & $\sigma$ \\
\hline $\mathrm{T} 1$ & $10,64 \pm$ & 0,10 & $2,51 \pm$ & 0,17 & 17,13 & \pm & 0,11 & 1,92 & \pm & 0,07 \\
\hline $\mathrm{T} 2$ & $8,93 \pm$ & 0,09 & $2,25 \pm$ & 0,10 & 13,26 & \pm & 0,09 & 0,44 & \pm & 0,01 \\
\hline $\mathrm{T} 3$ & $10,29 \pm$ & 0,34 & $4,86 \pm$ & 0,20 & 15,69 & \pm & 2,30 & 3,74 & \pm & 0,17 \\
\hline $\mathrm{T} 4$ & $12,72 \pm$ & 0,47 & $18,09 \pm$ & 0,23 & 41,68 & \pm & 0,48 & 2,75 & \pm & 0,03 \\
\hline T5 & $9,78 \pm$ & 0,09 & $2,38 \pm$ & 0,14 & 15,19 & \pm & 0,10 & 1,18 & \pm & 0,04 \\
\hline T6 & $10,47 \pm$ & 0,22 & $3,68 \pm$ & 0,18 & 16,41 & \pm & 1,21 & 2,83 & \pm & 0,12 \\
\hline $\mathrm{T} 7$ & $11,68 \pm$ & 0,28 & $10,30 \pm$ & 0,20 & 29,41 & \pm & 0,29 & 2,34 & \pm & 0,05 \\
\hline $\mathrm{T} 8$ & $9,61 \pm$ & 0,21 & $3,56 \pm$ & 0,15 & 14,47 & \pm & 1,20 & 2,09 & \pm & 0,09 \\
\hline T9 & $10,82 \pm$ & 0,28 & $10,17 \pm$ & 0,16 & 27,47 & \pm & 0,28 & 1,60 & \pm & 0,02 \\
\hline $\mathrm{T} 10$ & $11,51 \pm$ & 0,40 & $11,47 \pm$ & 0,21 & 28,68 & \pm & 1,39 & 3,24 & \pm & 0,10 \\
\hline
\end{tabular}

Os teores de umidade dos tratamentos variaram de 8,93 a 12,72\%, conforme apresentado na Tabela 3. O gradiente de umidade da madeira varia conforme espécie, características anatômicas e químicas e variações ambientais. Recomenda-se, para a fabricação de briquetes, que o teor de umidade do material esteja entre 8 e $15 \%$ de umidade, sendo assim, os valores de umidade obtidos estão de acordo com o recomendado na literatura [10]. Quanto maior o teor de umidade, maior será o consumo de energia necessário para a evaporação da água presente na biomassa nos processos de conversão energética, reduzindo a sua eficiência [6]. Por essa razão foi necessário secar as partículas dos resíduos ao ar livre, visando aumentar o seu potencial calorífico.

Com relação a composição química da biomassa, os maiores valores de extrativos e lignina foram encontrados nos tratamentos que continham resíduos da classe 4 (T4, T7, T9 e T10), o que já era esperado por se tratar de um material composto apenas por casca da espécie Pinus taeda. Segundo Colodette [18], de maneira geral, a casca apresenta diferenças químicas em comparação com a madeira, entre elas: maiores teores de extrativos e cinzas e menores teores de holocelulose. Dessa forma, estes tratamentos apresentam características químicas atrativas para serem utilizados como fonte de matéria prima na geração de energia.

O importante no caso dos extrativos é que são constituintes que ocupam espaços externos às paredes celulares (lúmens e vacúolos), logo eles somam peso seco às biomassas das paredes celulares. A lignina é o principal constituinte responsável pelo aumento do poder calorífico, pois ela possui entre 60 a $64 \%$ de carbono elementar em sua composição molecular, enquanto a celulose e as hemiceluloses possuem bem menos (42 a 46\%) [6].Quanto maior for o teor de lignina nas amostras, menor será a quantidade de materiais voláteis produzidos, pois como a lignina tem anel aromático, possui consequentemente maior quantidade de carbono fixo. Além disso, o tipo de lignina, assim como a relação siringila/guaiacila, influencia na geração de energia de uma biomassa [17]. A lignina guaiacila, presente em madeiras de coníferas, possui o C5 disponível para reação com outros anéis de fenilpropano, tem maior massa molecular e, consequentemente, é mais favorável à produção de carvão vegetal, devido à sua maior estabilidade térmica [19].

Conforme os dados apresentados na Tabela 4, pode-se observar que os tratamentos 4 e 10 resultaram nos maiores valores de poder calorífico, devido a presença do maior percentual de lignina e extrativos totais na sua composição química (Tabela 2). A lignina apresenta um conteúdo de carbono cerca de $50 \%$ maior do que o encontrado nos polissacarídeos, portanto apresenta um potencial realmente grande para produção de energia [20]. Além disso, os extrativos voláteis são importantes na queima direta da madeira, pois se degra- 
dam mais rapidamente e ajudam a manter a chama de combustão. Quirino et al. [21] concluíram que o poder calorífico da madeira, além da umidade, é influenciado pela constituição química da madeira, principalmente a lignina e extrativos (resinas, óleos-resinas, matérias graxas, óleos etc). Desta maneira, as coníferas que apresentam um conteúdo de resinas e lignina maior que as latifoliadas ostentam, consequentemente, um maior poder calorífico, que varia também conforme a parte da árvore que esteja em combustão (casca, nós, ramos, madeira do toco).

As densidades aparentes dos briquetes obtidas nesse estudo apresentaram resultados satisfatórios uma vez que o Banco de Dados de Biomassa do Brasil estipula uma densidade aparente de briquetes de 1000 $\mathrm{Kg} / \mathrm{m}^{3}$ a $1300 \mathrm{Kg} / \mathrm{m}^{3}$. Como podemos observar na Tabela 4, as densidades aparentes dos briquetes foram influenciadas pelas classes de resíduos utilizadas para sua fabricação, podendo-se verificar que os briquetes mais densos foram obtidos com resíduos florestais dos tratamentos que utilizaram os resíduos da Classe 4 (casca de pinus), pela melhor compactação promovida por esse material, o que acarretou na redução do volume do briquete para uma mesma massa. Com relação a resistência mecânica a compressão, os valores obtidos para os diferentes tratamentos variaram de 392 a $654 \mathrm{~kg}$ e não diferiram estatisticamente.

Tabela 4: Teste Tukey de comparação de médias e valores médios das características dos briquetes analisadas.

\begin{tabular}{c|cc|cc|cc}
\hline TRATAMENTOS & $\begin{array}{c}\text { PODER CALORIFICO } \\
\text { SUPERIOR } \\
\text { (MJ KG }^{-1} \text { ) }\end{array}$ & $\begin{array}{c}\text { DENSIDADE } \\
\text { APARENTE } \\
\text { (KG M }^{-3} \text { ) }\end{array}$ & $\begin{array}{c}\text { RESISTÉNCIA A } \\
\text { COMPRESSÃO } \\
\text { (KGF) }\end{array}$ \\
\hline $\mathrm{T} 1$ & $18,45 \pm 0,27$ & bc & $1.073,11 \pm 10,5$ & d & $524,00 \pm 136,9$ & a \\
T2 & $17,51 \pm 0,09$ & de & $1.147,12 \pm 18,9$ & abcd & $654,00 \pm 89,4$ & a \\
T3 & $18,27 \pm 0,24$ & bc & $1.075,04 \pm 17,6$ & d & $439,33 \pm 15,1$ & a \\
T4 & $19,39 \pm 0,23$ & a & $1.226,87 \pm 15,8$ & a & $418,67 \pm 230,9$ & a \\
T5 & $17,45 \pm 0,18$ & de & $1.157,67 \pm 17,6$ & abc & $573,33 \pm 45,0$ & a \\
T6 & $18,00 \pm 0,32$ & cd & $1.110,02 \pm 25,9$ & cd & $586,00 \pm 110,0$ & a \\
T7 & $18,36 \pm 0,06$ & bc & $1.183,07 \pm 25,2$ & abc & $472,00 \pm 50,0$ & a \\
T8 & $17,42 \pm 0,22$ & de & $1.131,93 \pm 69,9$ & bcd & $610,00 \pm 15,6$ & a \\
T9 & $17,35 \pm 0,20$ & e & $1.201,17 \pm 18,1$ & ab & $581,67 \pm 40,1$ & a \\
T10 & $18,83 \pm 0,12$ & ab & $1.176,06 \pm 16,6$ & abc & $392,00 \pm 62,2$ & a \\
\hline
\end{tabular}

Na Tabela 5 são apresentados os valores obtidos dos poderes caloríficos superior, inferior e útil e de densidade energética dos briquetes produzidos com as diferentes classes de resíduos florestais.

Tabela 5: Valores médios dos poderes caloríficos superior, inferior e útil e densidade energética dos briquetes produzidos pelos diferentes tratamentos.

\begin{tabular}{c|c|c|c|c}
\hline TRATAMENTO & PCS & PCI & PCU & $\begin{array}{c}\text { DE } \\
\text { (Mcal. }^{-3} \text { ) }\end{array}$ \\
\hline T1 & 4406,8 & 3874,0 & 3461,3 & 3714,4 \\
\hline T2 & 4181,7 & 3754,9 & 3419,4 & 3922,4 \\
\hline T3 & 4364,0 & 3853,0 & 3456,1 & 3715,5 \\
\hline T4 & 4631,9 & 3966,4 & 3461,5 & 4246,8 \\
\hline T5 & 4167,1 & 3700,7 & 3338,3 & 3864,6 \\
\hline T6 & 4298,4 & 3785,7 & 3389,1 & 3761,9 \\
\hline T7 & 4385,9 & 3803,5 & 3358,8 & 3973,7 \\
\hline T8 & 4161,2 & 3703,7 & 3347,4 & 3789,0 \\
\hline T9 & 4143,7 & 3630,2 & 3236,9 & 3888,1 \\
\hline T10 & 4497,1 & 3910,6 & 3460,2 & 4069,4 \\
\hline
\end{tabular}

PCI = Poder Calorífico Inferior, PCS = Poder Calorífico Superior, PCU = Poder Calorífico Útil, expressos em $\mathrm{kcal} / \mathrm{kg} ; \mathrm{DE}=$ densidade energética expressa em $\mathrm{Mcal} / \mathrm{m}^{3}$

Os briquetes produzidos com classe de resíduos 4 (casca de Pinus taeda) resultaram nos mais altos valores para densidade energética (tratamentos 4, 7, 9 e 10), explicado devido ao alto teor de lignina e de extra- 
tivos totais encontrado para esse resíduo. Na Tabela 6 é apresentada a matriz de correlação entre as variáveis originais analisadas referente a qualidade dos briquetes. As duas primeiras componentes explicaram aproximadamente $93 \%$ da variância total dos dados. Optou-se no presente estudo por considerar apenas duas componentes principais devido à grande parcela da variância explicada por elas.

As maiores correlações ocorreram entre as variáveis extrativos totais, lignina e densidade energética. O determinante da matriz foi $>0$, indicando que a matriz de correlação é diferente de uma matriz identidade. Portanto, a técnica de componentes principais é viável. O valor do Teste KMO (Medida Kaiser-Meyer-Olkin) foi de 0,538, indicando que a análise de componentes principais foi adequada, pois segundo Hair et al. [22], valores aceitáveis variam entre 0,5 a 1,0. Essa adequação é comprovada também pelo Teste de Esfericidade de Bartlett, que foi significante $(\mathrm{p}<0,05)$. A Tabela 7 apresenta os autovetores das duas primeiras componentes principais obtidas por meio da matriz de correlação das variáveis originais, a variância explicada acumulada e autovalores.

Tabela 6: Matriz da correlação de Pearson das características das biomassas e a qualidade dos briquetes.

\begin{tabular}{c|cccccccc}
\hline & TU & EXT & TL & TC & PC & DA & RC & DE \\
\hline TU & 1 & 0,901 & 0,931 & 0,552 & 0,831 & 0,495 & $-0,796$ & 0,833 \\
EXT & & 1 & 0,985 & 0,394 & 0,688 & 0,774 & $-0,678$ & 0,933 \\
TL & & & 1 & 0,322 & 0,702 & 0,766 & $-0,655$ & 0,940 \\
TC & & & & 1 & 0,630 & $-0,198$ & $-0,776$ & 0,228 \\
PC & & & & & 1 & 0,183 & $-0,863$ & 0,723 \\
DA & & & & & & 1 & $-0,134$ & 0,795 \\
RC & & & & & & & 1 & $-0,625$ \\
DE & & & & & & & & 1 \\
\hline
\end{tabular}

TU: teor de umidade; EXT: extrativos totais; TL: lignina Klason; PC: poder calorífico; DA: Densidade Aparente; RC: Resistência à compressão; DE: Densidade energética

Tabela 7: Autovetores das duas primeiras componentes principais.

\begin{tabular}{|c|c|c|}
\hline \multirow{2}{*}{ PARÂMETROS } & \multicolumn{2}{|c|}{ COMPONENTES } \\
\hline & 1 & 2 \\
\hline TU - Teor de Umidade (\%) & 0,960 & $-0,078$ \\
\hline EXT - Teor de extrativos totais (\%) & 0,962 & 0,217 \\
\hline TL - Teor de lignina Klason $(\%)$ & 0,959 & 0,249 \\
\hline TC - Teor de cinza $(\%)$ & 0,531 & $-0,761$ \\
\hline PC - Poder calorífico superior $\left(\mathrm{MJ} \mathrm{kg}^{-1}\right)$ & 0,847 & $-0,373$ \\
\hline DA - Densidade aparente $\left(\mathrm{kg} \mathrm{m}^{-3}\right)$ & 0,610 & 0,767 \\
\hline RC - Resistência à compressão (kgf) & $-0,823$ & 0,490 \\
\hline DE - Densidade energética $\left(\mathrm{Mcal} \mathrm{m}^{3}\right)$ & 0,927 & 0,306 \\
\hline Variância explicada (\%) & 70,932 & 21,950 \\
\hline Variância explicada acumulada (\%) & 70,932 & 92,882 \\
\hline Autovalores & 5,675 & 1,756 \\
\hline
\end{tabular}

As variáveis EXT, TU, TL, DE, PC e RC apresentaram maior correlação com o primeiro componente principal, indicando que são as variáveis mais importantes na análise. Pois este componente representa 70,932 \% da variância total explicada. As variáveis TC e DA foram mais correlacionadas com o segundo componente principal, que representa apenas $21,95 \%$ da variância. Portanto, a primeira componente principal pode ser considerada um índice de desempenho global para a avaliação dos briquetes lignocelulósicos, onde os mais elevados coeficientes da componente principal, em módulo, foram relativos aos teores de extrativos, de umidade, de lignina e a densidade energética [23]. Pode-se inferir que essa componente reúne as melhores características para avaliação preliminar dos briquetes produzidos por materiais lignocelulósicos visando o seu uso bioenergético. Na Figura 1, encontra-se o diagrama de ordenação das variáveis originais e 
os escores das duas primeiras componentes principais.

Na Figura 1, pode-se observar que as variáveis mais correlacionadas com o primeiro componente principal (EXT, TU, TL, DE, PC) estão localizadas a direita do gráfico, exceto a variável RC, que se localiza a esquerda, por possuir valores negativos. Os briquetes produzidos puramente das classes 3 e 4 (tratamentos 3 e 4) e a mistura das duas classes de resíduos (tratamento 10) formaram grupos distintos. Além disso, o tratamento 4 se destacou dentro do componente 1, por seus elevados teores de extrativos e lignina; já os tratamentos 3 e 10 tiveram destaque no componente 2, devido aos maiores teores de cinzas.

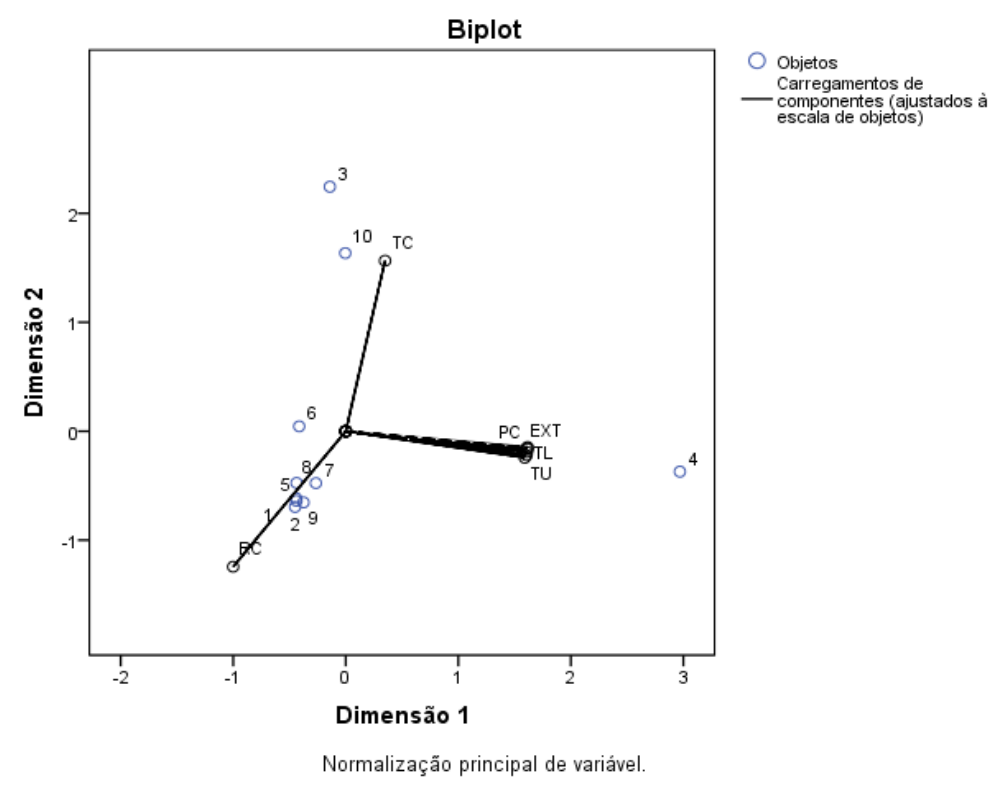

Figura 1: Diagrama de ordenação dos tratamentos avaliados considerando os escores e autovetores das componentes principais 1 e 2. Em que: TU= teor de umidade, $\mathrm{EXT}=$ teor de extrativos totais, $\mathrm{TL}=$ teor de lignina Klason, $\mathrm{TC}=$ teor de cinzas, $\mathrm{PC}$ = poder calorífico superior, $\mathrm{DA}=$ densidade aparete, $\mathrm{RC}=$ resistência a compressão, $\mathrm{DE}=$ densidade energética, 1 a $10=$ Tratamentos

\section{CONCLUSÕES}

Os resíduos florestais apresentaram características energéticas favoráveis, indicando o potencial desse material para uso como fonte de energia, com destaque para a classe de resíduos 4 (casca de Pinus taeda), que resultou nos maiores valores de lignina. $\mathrm{O}$ briquetes produzidos apresentaram resultados satisfatórios quanto a teor de umidade e densidade aparente recomendados, sendo os tratamentos com os resíduos florestais de casca de pinus resultaram nos maiores valores de densidades aparente e energéticas.

\section{AGRADECIMENTOS}

Os autores agradecem a empresa Celulose Riograndense (CMPC) pela disponibilidade do material vegetal utilizado nesse estudo. Também aos órgãos de fomento CAPES e CNPq.

\section{BIBLIOGRAFIA}

[1] SAAD, S.A., ISA, K.M., BAHARI, R. "Chemically modified sugarcane bagasse as a potentially low-cost biosorbent for dye removal”, Desalination, v. 264, n. 1-2, pp. 123-128, dez. 2010.

[2] Ministério de Minas e Energia - MME. "Biomassa é a segunda maior fonte de energia em 2016", http://www.mme.gov.br/web/guest/todas-as-noticias/-/asset_publisher/pdAS9IcdBICN/content/biomassa-e-asegunda-maior-fonte-de-energia-em-

2016?inheritRedirect=false\&redirect=http $\% 3 \mathrm{~A} \% 2 \mathrm{~F} \% 2 \mathrm{Fwww} . \mathrm{mme}$.gov.br\%2Fweb\%2Fguest $\% 2$ Ftodas-asnotici-

as\%3Fp_p_id\%3D101_INSTANCE_pdAS9IcdBICN\%26p_p_lifecycle\%3D0\%26p_p_state\%3Dnormal\%26 p_p_mode\%3Dview\%26p_p_col_id\%3Dcolumn-

1\%26p_p_col_count\%3D1\%26_101_INSTANCE_pdAS9IcdBICN_cur\%3D30\%26_101_INSTANCE_pdAS 
9IcdBICN_keywords\%3D\%26_101_INSTANCE_pdAS9IcdBICN_advancedSearch\%3Dfalse\%26_101_INS TAN-

CE_pdAS9IcdBICN_delta\%3D30\%26p_r_p_564233524_resetCur\%3Dfalse\%26_101_INSTANCE_pdAS9I cdBICN_andOperator\%3Dtrue.

Acessado em setembro de 2017.

[3] BENEDETTI, O.I.S., CHAVES, R.Q., MAGALHÃES, A.M. et al., “Análise preliminar da produção de etanol a partir de celulose: caminhos e desafios para a produção de álcool no Rio Grande Do Sul”, Engenharia Ambiental, v. 6, n. 2, pp. 272-284, mai/ago 2009.

[4] BERNI, M. D. O PAPEL, https://www.celuloseonline.com.br/eficiencia-energetica-mauro-donizetiberni-fracionamento-da-biomassa-lignocelulosica-com-finalidade-de-produzir-novos-produtos/. Acessado em agosto de 2017.

[5] BOUNDELLE, G.M., CHIES, D., MARTINS, D.G., “O processo de fabricação de painéis compensados no Estado do Paraná analisado por meio dos rendimentos e dos resíduos gerados", In: Anais do Congresso Ibero-Americano De Pesquisa E Desenvolvimento De Produtos Florestais, Curitiba, 2002.

[6] FOELKEL, C.E.B., "Utilização da Biomassa do Eucalipto para Produção de Calor, Vapor e Eletricidade Parte 1: Biomassa Florestal \& Florestas Energéticas”, Eucalyptus Online Book \& Newsletter, 2016.

[7] FELFLI, F.F, LUENGO, C.A., ROCHA, J.D., "Briquetes torrificados: viabilidade técnico-econômica e perspectivas no mercado brasileiro", In: Encontro de Energia no Meio Rural, Campinas, 2004.

[8] ORRELANA, B.B.M.A. Utilização de resíduos de biomassa do Distrito Federal para fins energéticos, Tese de D.Sc.,UnB, Brasília, DF, Brasil, 2019.

[9] PONTE, M.R., GADELHA, A.M.T., MACHADO, Y.L., et al. "Blendas de bagaço de cana-de-açúcar, podas de mangueira e cajueiro: caracterização das propriedades e investigação de seus potenciais energéticos”, Revista Matéria, v. 24, n. 2, 2019.

[10] PAULA, L.E.R., "Produção e avaliação de briquetes de resíduos lignocelulósicos", Dissertação de M.Sc., UFLA, Lavras, MG, Brasil, 2010.

[11] TAPPI. T $204 \mathrm{~cm}-97$. Solvent extractives of wood and pulp, 1997.

[12] TAPPI. T $210 \mathrm{~cm}-97$. Sampling and testing wood pulp shipments for moisture, 1997.

[13] TAPPI. T 264 cm-97. Preparation of Wood for Chemical Analysis, 1997.

[14] TAPPI. T222 om-02. Acid-insolub lignin in wood and pulp, 2002.

[15] TAPPI. T 211 om-02. Ash in wood, pulp, paper and paperboard: combustion at 525 2002.

[16] SILVA, D. “Avaliação da eficiência energética em uma indústria de painéis compensados”, Tese de D.Sc., UFPR, Curitiba. 2001.

[17] GOUVÊA, A.F.G., "Produção de briquete a partir da adição da lignina kraft com resíduo da indústria moveleira", Tese de D.Sc., UFV, Viçosa, MG, Brasil, 2012.

[18] COLODETTE, J.L. “Caderno didático de química da madeira”, Curso de Pós-graduação Strictu Sensu em Tecnologia de Celulose e Papel, Viçosa, MG, Brasil, 2001.

[19] CASTRO, A.F.N.M., "Efeito da idade e de materiais genéticos de Eucalyptus sp. na madeira e carvão vegetal”, Dissertação de M.Sc. UFV, Viçosa, MG, Brasil, 2011.

[20] VITAL, B.R., CARNEIRO, A.C.O., PEREIRA, B.L.C., "Qualidade da madeira para fins energéticos", In: Santos, F., Colodette, J., Queiroz, J. H. (Org.), Bioenergia \& Biorrefinaria: Cana-de-Açúcar \& Espécies Florestais, 1 ed., pp. 321-354, Visconde do Rio Branco, Suprema, 2013.

[21] QUIRINO, W. F., VALE, A.M., ANDRADE, A.P.A. et al., "Poder calorífico da madeira e de materiais lignocelulósicos", Revista da Madeira, n.89, pp.100-106, 2005.

[22] HAIR J.F., ANDERSON R.E., TATHAM, R.L., Multivariate data analysis with readings, 2 ed, New York, Macmillan Publishing Company, 1987.

[23] DIAS JÚNIOR, A.F., ANDRADE, A.M., CARVALHO, A.M. et al., "Produção de briquetes de moinha de carvão vegetal e resíduos lignocelulósicos visando uso bioenergético", Scientia Forestalis, v. 44, n. 110, pp. 453-462, jun. 2016. 


\section{ORCID}

Angelo Fernando de Oliveira Saccol

https://orcid.org/0000-0003-1851-5216

Carline Andréa Welter

https://orcid.org/0000-0003-2690-2881

Rossana Cortelini da Rosa

https://orcid.org/0000-0002-5754-7918

Rodrigo Coldebella

https://orcid.org/0000-0002-7063-1874

Solon Jonas Longhi

https://orcid.org/0000-0002-5701-2139

Jorge Antonio de Farias

https://orcid.org/0000-0001-7494-4176

Cristiane Pedrazzi

https://orcid.org/0000-0003-4976-467X 